\title{
Algebraic properties of an integrable $t-J$ model with impurities
}

\author{
Angela Foerster ${ }^{\mathrm{a}, 1}$, Jon Links ${ }^{\mathrm{a}, \mathrm{b}}$, Arlei Prestes Tonel ${ }^{\mathrm{a}}$ \\ a Instituto de Física da UFRGS, Av. Bento Gonçalves 9500, Porto Alegre, RS, Brazil \\ ${ }^{b}$ Department of Mathematics, University of Queensland, Queensland, 4072, Australia
}

Received 25 January 1999; accepted 17 March 1999

\begin{abstract}
We investigate the algebraic structure of a recently proposed integrable $t-J$ model with impurities. Three forms of the Bethe ansatz equations are presented corresponding to the three choices for the grading. We prove that the Bethe ansatz states are highest weight vectors of the underlying $g l(2 \mid 1)$ supersymmetry algebra. By acting with the $g l(2 \mid 1)$ generators we construct a complete set of states for the model. (c) 1999 Elsevier Science B.V. All rights reserved.
\end{abstract}

\section{Introduction}

The recent interest in the study of impurities coupled to both Fermi and Luttinger liquids has led to the introduction of new theoretical approaches in order to study such systems. One approach is to adopt the prescription of the Quantum Inverse Scattering Method (QISM) which permits impurities to be incorporated into a model in such a way that integrability is not violated. The origins of this method trace back to the work of Andrei and Johannesson [1] who constructed an integrable version of a spin 1/2 Heisenberg chain containing an arbitrary spin $S$ impurity (see also Refs. [2,3]).

The long studied $t-J$ model describes strongly correlated electrons interacting on a lattice comprising nearest neighbour hopping and spin exchange interactions. The model is defined on a restricted Hilbert space such that double occupancy of electrons at a

\footnotetext{
${ }^{1}$ Corresponding author. E-mail:angela@if.ufrgs.br 
single site is forbidden. On a one-dimensional lattice with periodic boundary conditions there exists a special choice of $t, J$ such that the model can be solved exactly [4-6]. Subsequently, it was shown that at this point the model acquires supersymmetry with respect to the Lie superalgebra $g l(2 \mid 1)$ [7]. The supersymmetry reflects the fact that the model possesses both bosonic and fermionic degrees of freedom. The existence of solvability and supersymmetry can be understood by the fact that the model can be derived in the context of the QISM [8,9] which also proves integrability. Application of the algebraic Bethe ansatz leads to three different sets of Bethe ansatz equations corresponding to different choices of the grading. Furthermore, theoretical techniques based on the underlying supersymmetry expose significant consequences for the multiplet structure of the model. Specifically, it was shown in [9] that the Bethe states are highest weight states of the $g l(2 \mid 1)$ superalgebra and that a complete set of states could be obtained by exploiting this supersymmetry.

Adopting the QISM, Bedürftig et al. [10] introduced an integrable periodic $t-J$ model with impurity. In this model, the nature of the impurity is such that doubly occupied states can occur at those sites at which the impurities are situated. The origin of the impurity comes from changing the representation of the $g l(2 \mid 1)$ generators at some lattice sites from the fundamental three-dimensional representation to the one parameter family of typical four dimensional representations which were introduced in [11] to derive the supersymmetric $U$ model. The resulting impurity model is significant in that the parameters from the four-dimensional representations can be varied continuously without losing integrability. The impurities couple to both spin and charge degrees of freedom and these parameters describe the strength of the coupling of the impurities to the host chain. Limiting cases describe effective supersymmetric $t-J$ models with additional sites or decoupled impurities in a $t-J$ chain with twisted boundary conditions. Using the method of the algebraic Bethe ansatz, a solution for a particular choice of grading was derived. Also, a comprehensive analysis of thermodynamic properties such as ground state structure, elementary excitations, low-temperature specific heat and transport properties was given in [10].

In this paper, we undertake an investigation into the detailed algebraic aspects of this impurity model. First, we present all three forms of the algebraic Bethe ansatz solutions corresponding to the three different choices of grading, viz. FBB, BBF, BFB. The case of FBB is that given in [10]. A significant point here is that only recently have new Bethe ansatz methods been proposed in order to solve the cases of the other gradings because of a lack of a suitable (unique) reference state. Rather one is forced to work with a subspace of reference states. This approach was developed in the works of Abad and Ríos for an $S U(3)$ impurity spin chain [12,13] and has already been adopted in [14] to find a Bethe ansatz solution of the supersymmetric $U$ model starting from a ferromagnetic space of states and in Refs. [15-17] regarding another integrable $t-J$ model with impurities. At this point we mention that other impurity $t-J$ models have been studied. In the work of Bares impurities were introduced into the model by way of inhomogeneities in the transfer matrix of the system [18]. Kondo (magnetic) impurities for arbitrary spin $S$ have been studied by Schlottmann and Zvyagin [19]. These works 
treat cases of bulk impurities with periodic boundary conditions. It is also possible to consider open chains where the impurities are situated on the boundary. In these cases the impurity interactions assume very different forms than the case of bulk impurities and examples of these models have been considered in works of Wang et al. [20] and Zhou et al. [21].

We also show that the Bethe ansatz states are highest weight states with respect to the underlying $g l(2 \mid 1)$ supersymmetry algebra. By acting with the $g l(2 \mid 1)$ lowering operators on the Bethe states we obtain additional eigenvectors. Then by a combinatorial argument we claim that in fact we recover a complete set of states for the model. Aside from the (non-impurity) integrable $t-J$ model, completeness of the Bethe ansatz solution has been studied for example for the cases of the Heisenberg model [22,23], the Hubbard model [24] and the $g l(2 \mid 2)$ supersymmetric extended Hubbard model [25]. Completeness for the integrable spin $S$ impurity Heisenberg model was discussed by Kirillov [26]. Our results give the first example of completeness for a supersymmetric, integrable impurity model.

The paper is organized as follows. In Section 2 we recall the construction of the model through the QISM and also fix notation. In Section 3 we discuss the algebraic Bethe ansatz solutions of this model. The highest weight property of the Bethe states is demonstrated in Section 4 and the completeness of the Bethe states is shown in Section 5.

\section{Quantum inverse scattering method}

Recall that the Lie superalgebra $g l(2 \mid 1)$ has generators $\left\{E_{j}^{i}\right\}_{i, j=1}^{3}$ satisfying the commutation relations

$$
\left[E_{j}^{i}, E_{l}^{k}\right]=\delta_{j}^{k} E_{l}^{i}-(-1)^{(\mid i]+|j|)(|k|+|l|)} \delta_{l}^{i} E_{j}^{k},
$$

where the $\mathbb{Z}_{2}$-grading on the indices is determined by

$$
\begin{array}{ll}
{[i]=0} & \text { for } i=1,2, \\
{[i]=1} & \text { for } i=3 .
\end{array}
$$

This induces a $\mathbb{Z}_{2}$-grading on the $g l(2 \mid 1)$ generators through

$$
\left[E_{j}^{i}\right]=([i]+[j])(\bmod 2) \text {. }
$$

The vector module $V$ has basis $\left\{v^{i}\right\}_{i=1}^{3}$ with action defined by

$$
E_{j}^{i} v^{k}=\delta_{j}^{k} v^{i}
$$

Associated with this space there is a solution $R(u) \in \operatorname{End}(V \otimes V)$ of the Yang-Baxter equation

$$
R_{12}(u-v) R_{13}(u) R_{23}(v)=R_{23}(v) R_{13}(u) R_{12}(u-v)
$$


on the space $V \otimes V \otimes V$ which is given by

$$
R(u)=I \otimes I-\frac{2}{u} \sum_{i, j} e_{j}^{i} \otimes e_{i}^{j}(-1)^{[j]} .
$$

We remark that Eq. (3) is acting on a supersymmetric space so the multiplication of tensor products is governed by the relation

$$
(a \otimes b)(c \otimes d)=(-1)^{|b|[c \mid} a c \otimes b d
$$

for homogeneous operators $b, c$.

The solution given by Eq. (4) allows us to construct a universal $L$-operator which reads

$$
L(u)=I \otimes I-\frac{2}{u-\lambda} \sum_{i, j} e_{j}^{i} \otimes E_{i}^{j}(-1)^{[j]}
$$

for any complex parameter $\lambda$. This operator gives us a solution of the Yang-Baxter equation of the form

$$
R_{12}(u-v) L_{13}(u) L_{23}(v)=L_{23}(v) L_{13}(u) R_{12}(u-v)
$$

on the space $V \otimes V \otimes g l(2 \mid 1)$ which follows from the commutation relations Eq. (1). We use the one-parameter family of four-dimensional representations of $g l(2 \mid 1)[11]$ acting on the module $W$ with basis $\left\{v^{a}, v^{b}, v^{c}, v^{d}\right\}$. The explicit matrix representatives are given by

$$
\begin{aligned}
& E_{2}^{1}=|b\rangle\langle c|, \\
& E_{1}^{2}=|c\rangle\langle b|, \\
& E_{1}^{1}=-|c\rangle\langle c|-| d\rangle\langle d|, \\
& E_{2}^{2}=-|b\rangle\langle b|-| d\rangle\langle d| \\
& E_{3}^{2}=\sqrt{\alpha}|a\rangle\langle b|+\sqrt{\alpha+1}| c\rangle\langle d|, \\
& E_{2}^{3}=\sqrt{\alpha}|b\rangle\langle a|+\sqrt{\alpha+1}| d\rangle\langle c|, \\
& E_{3}^{1}=-\sqrt{\alpha}|a\rangle\langle c|+\sqrt{\alpha+1}| b\rangle\langle d|, \\
& E_{1}^{3}=-\sqrt{\alpha}|c\rangle\langle a|+\sqrt{\alpha+1}| d\rangle\langle b|, \\
& E_{3}^{3}=\alpha|a\rangle\langle a|+(\alpha+1)(|b\rangle\langle b|+| c\rangle\langle c|)+(\alpha+2)| d\rangle\langle d| .
\end{aligned}
$$

This representation holds for any complex value of $\alpha$. However, in the following we will assume that $\alpha$ is real and positive ensuring that the Hamiltonian is Hermitian. By taking this representation in the expression (6) we obtain an $R$-matrix, denoted by $R^{*}(u)$, which satisfies the following Yang-Baxter equation:

$$
R_{12}(u-v) R_{13}^{*}(u) R_{23}^{*}(v)=R_{23}^{*}(v) R_{13}^{*}(u) R_{12}(u-v)
$$

acting on $V \otimes V \otimes W$. 
The impurity model is constructed with generic quantum spaces represented by $V$ and the impurity spaces $W$. To this end take some index set $I=\left\{p_{1}, p_{2}, \ldots, p_{l}\right\}, 1 \leqslant p_{i} \leqslant L$ and define

$$
X=\bigotimes_{i=1}^{L} X_{i},
$$

where

$$
\begin{array}{ll}
X_{i}=V & \text { if } i \notin I, \\
X_{i}=V \otimes W & \text { if } i \in I .
\end{array}
$$

In other words, for each $i \in I$ we are coupling an impurity into the lattice which will be situated between the sites $i$ and $i+1$.

Next we define the monodromy matrix

$$
T(u)=\bar{R}_{01}(u) \bar{R}_{02}(u) \ldots \bar{R}_{0 L}(u),
$$

where we have

$$
\begin{array}{ll}
\bar{R}_{0 i}(u)=R_{0 i}(u) & \text { for } i \notin I, \\
\bar{R}_{0 i}(u)=R_{0 i^{\prime}}(u) R_{0 i^{\prime \prime}}^{*}(u-\lambda) & \text { for } i \in I
\end{array}
$$

and we set $\lambda=\alpha+2$. Above, the indices $i^{\prime}$ and $i^{\prime \prime}$ refer to the two spaces in $X_{i}$ (cf. Eq. (9)). A consequence of Eqs. (3), (8) is that the monodromy matrix satisfies the intertwining relation

$$
R_{12}(u-v) T_{13}(u) T_{23}(v)=T_{23}(v) T_{13}(u) R_{12}(u-v)
$$

acting on the space $V \otimes V \otimes X$. The transfer matrix is defined by

$$
\tau(u)=\operatorname{tr}_{0} \sigma_{0} T(u),
$$

where the matrix $\sigma$ has entries

$$
\sigma_{j}^{i}=(-1)^{[i][j]} \delta_{j}^{i}
$$

from which the Hamiltonian [10] is obtained through

$$
H=-\left.2 \frac{d}{d u} \ln \left(v^{L} \tau(u)\right)\right|_{u=0} .
$$

From Eq. (10) it is concluded by the usual arguments that the transfer matrix provides a set of abelian symmetries for the model and hence the Hamiltonian is integrable. In the next section we will solve the model by the algebraic Bethe ansatz approach. We use a different grading (BBF) from that adopted in [10] (FBB) in order to obtain the Bethe ansatz equations in a form that is more convenient to prove completeness of the model. We note that for the case of one impurity, the equivalence of these two forms of the Bethe ansatz equations has already been discussed in [10]. We also present the third form (BFB) of the Bethe ansatz equations. 


\section{Algebraic Bethe ansatz solution}

By a suitable redefinition of the matrix elements, the operators $R$ and $R^{*}$ may be written in terms of matrices which satisfy the Yang-Baxter equations (3), (8) without $\mathbb{Z}_{2}$-grading (see e.g. Ref. [27]). These matrices read

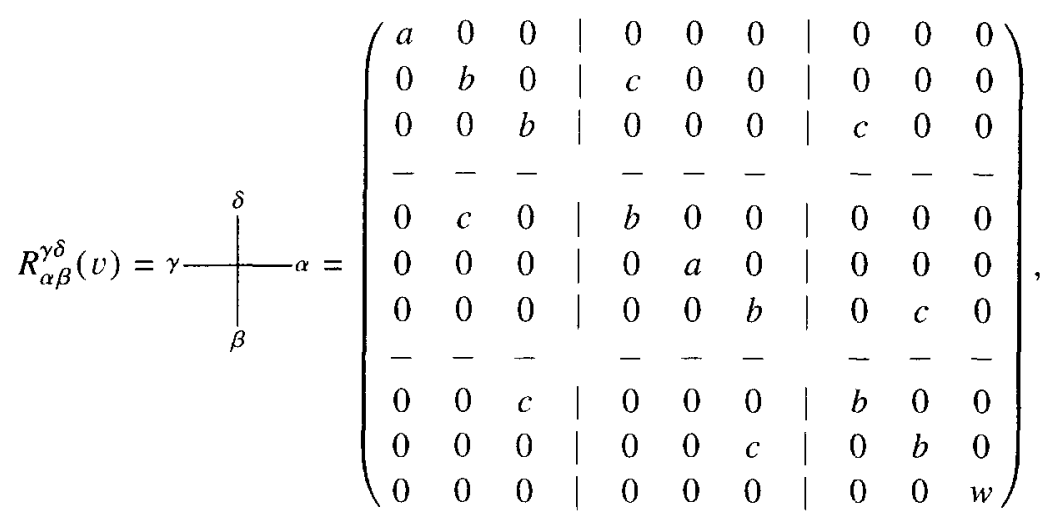

where $\alpha, \beta$ ( $\gamma$ and $\delta$ ) are column (row) indices running from 1 to 3 and

$$
a=1-2 / v, \quad b=1, \quad c=-2 / v, \quad w=-1-2 / v
$$

and

$$
\begin{aligned}
& R_{\alpha \beta}^{* \gamma \delta}(v)=\gamma{\frac{\delta}{\hat{\gamma}_{\beta}}}^{\alpha}
\end{aligned}
$$

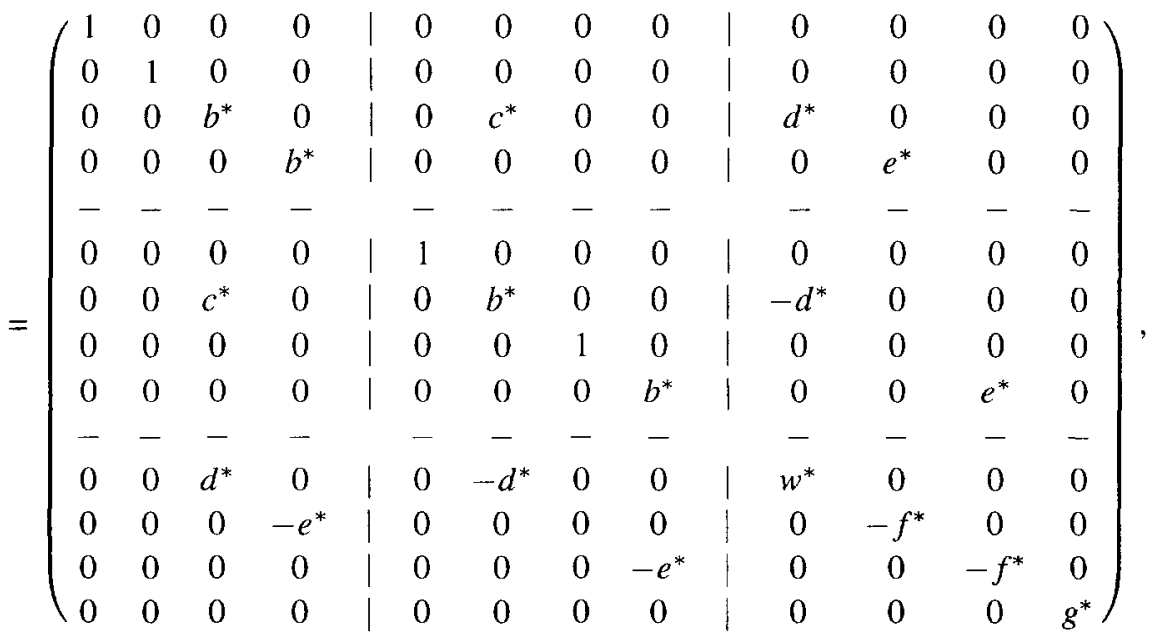

with

$$
b^{*}=1+2 / v^{\prime}, \quad c^{*}=-2 / v^{\prime}, \quad d^{*}=2 \sqrt{\alpha} / v^{\prime}, \quad e^{*}=-2 \sqrt{\alpha+1} / v^{\prime},
$$




$$
f^{*}=1+2(\alpha+1) / v^{\prime}, \quad w^{*}=1+2 \alpha / v^{\prime}, \quad g^{*}=1+2(\alpha+2) / v^{\prime}
$$

and $v^{\prime}=v-\alpha-2$. The $R$-matrix (13) acts in the tensor product of the two 3dimensional spaces $V \otimes V$ while $R^{*}$ (15) acts in the tensor product of $V \otimes W$, where $W$ is the 4-dimensional space. Hereafter we will use these forms.

Next we construct the monodromy matrix

$$
Y(u)=\left(\begin{array}{lll}
Y_{1}^{1}(u) & Y_{1}^{2}(u) & Y_{1}^{3}(u) \\
Y_{2}^{1}(u) & Y_{2}^{2}(u) & Y_{2}^{3}(u) \\
Y_{3}^{1}(u) & Y_{3}^{2}(u) & Y_{3}^{3}(u)
\end{array}\right)
$$

whose elements are operators acting in the quantum space $X$. The action of these elements is given by

$$
\pi\left(Y_{j}^{i}(u)\right)_{\left\{\beta^{\prime}\right\}}^{\{\beta\}}=(-1)^{\left.(\mid i]\left|\left\{\beta^{\prime}\right\}\right|+|j|\left|\left\{\beta^{\prime}\right\}\right|+[i]|\{\beta\}|\right)} T_{j\left\{\beta^{\prime}\right\}}^{i\{\beta\}}(u),
$$

such that $Y(u)$ satisfies the Yang-Baxter algebra without $\mathbb{Z}_{2}$-grading

$$
R_{12}(u-v) Y_{13}(u) Y_{23}(v)=Y_{23}(v) Y_{13}(u) R_{12}(u-v) .
$$

The transfer matrix is expressible in terms of this representation by

$$
\tau(u)=\sum_{i=1}^{3}(-1)^{\mid i]+[i||\{\beta\}]} \pi\left(Y_{i}^{i}(u)\right)_{\left\{\beta^{\prime}\right\}}^{\{\beta\}} .
$$

In the following we will omit the symbol $\pi$ for ease of notation.

For a given $\{\alpha\}=\left(\alpha_{1}, \alpha_{2}, \ldots, \alpha_{l}\right), \alpha_{i}=a, b$ we define the first pseudovacuum $v^{\{\alpha\}} \in X$ by

$$
v^{\{\alpha\}}=\bigotimes_{i=1}^{L} w^{i}
$$

where

$$
\begin{array}{ll}
w^{i}=v^{1} & \text { for } i \notin I, \\
w^{i}=v^{1} \otimes v^{\alpha_{j}} & \text { for } i=p_{j} \in I .
\end{array}
$$

An example for the case $L=10$ and $l=2$ is depicted below:

$$
\begin{aligned}
& v^{\{\alpha\}}=\mid \text { 年 }|| \mid \text { 年 }|||| \quad \alpha_{i}=a, b \\
& \begin{array}{llllllllllll}
1 & 1 & \alpha_{1} & 1 & 1 & 1 & 1 & \alpha_{2} & 1 & 1 & 1 & 1
\end{array}
\end{aligned}
$$

Setting

$$
S^{\{\beta\}}(\{u\})=Y_{1}^{\beta_{1}}\left(u_{1}\right) Y_{1}^{\beta_{2}}\left(u_{2}\right) \ldots Y_{1}^{\beta_{N}}\left(u_{N}\right), \quad \beta_{i}=2,3
$$


we look for a set of eigenstates of the transfer matrix of the form

$$
\Phi^{j}=\sum_{\{\beta, \alpha\}} S^{\{\beta\}}(\{u\}) v^{\{\alpha\}} F_{\{\beta, \alpha\}}^{j},
$$

where the $F_{\{\beta, \alpha\}}^{j}$ are undetermined coefficients. We appeal to the algebraic equations given by $\mathrm{Eq}$. (19) to determine the constraints on the variables $u_{i}$ needed to force Eq. (21) to be an eigenstate. Although many relations occur as a result of Eq. (19) only the following are required:

$$
\begin{aligned}
Y_{1}^{1}(v) Y_{1}^{\beta}(u) & =a(u-v) Y_{1}^{\beta}(u) Y_{1}^{1}(v)-b(u-v) T_{1}^{\beta}(v) Y_{1}^{1}(u), \\
Y_{\gamma}^{\gamma^{\prime}}(v) Y_{1}^{\alpha}(u) & =Y_{1}^{\alpha^{\prime}}(u) Y_{\gamma}^{\gamma^{\prime \prime}}(v) r_{\gamma^{\prime \prime} \alpha^{\prime}}^{\gamma^{\prime} \alpha}(v-u)-b(v-u) Y_{1}^{\gamma^{\prime}}(v) Y_{\gamma}^{\alpha}(u), \\
a(v-u) Y_{1}^{\alpha}(v) Y_{1}^{\beta}(u) & =Y_{1}^{\beta^{\prime}}(u) Y_{1}^{\alpha^{\prime}}(v) r_{\beta^{\prime} \alpha^{\prime}}^{\beta \alpha}(v-u) .
\end{aligned}
$$

All of the indices in Eqs. (22), (23), (24) assume only the values 2 and 3. Above, the matrix $r(u)$ arises as a submatrix of $R(u)$ and satisfies the Yang-Baxter equation for a $g l(1 \mid 1)$ invariant model.

Using Eq. (22) two types of terms arise when $Y_{1}^{1}$ is commuted through $Y_{1}^{\alpha}$. In the first type $Y_{1}^{l}$ and $Y_{1}^{\alpha}$ preserve their arguments and in the second type their arguments are exchanged. The first type of terms are called wanted terms because they will give a vector proportional to $\Phi^{j}$, and the second type are unwanted terms (u.t.). We find that

$$
Y_{1}^{1}(v) \Phi^{j}=a(v)^{L} \prod_{i=1}^{N} a\left(u_{i}-v\right) \Phi^{j}+\text { u.t. }
$$

where we have used the fact that

$$
Y_{1}^{1}(v) v^{\{\alpha\}}=a(v)^{L} v^{\{\alpha\}} .
$$

For the case of the previous example we have

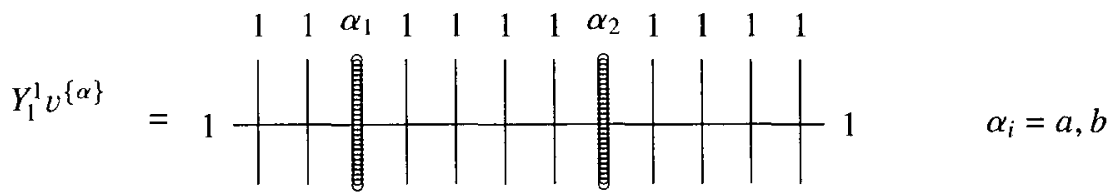

Similarly, for $i=2,3$ we have from Eq. (23) (no sum over $i$ )

$$
\begin{aligned}
Y_{i}^{i}(v) \Phi^{j} & =S^{\left\{\beta^{\prime}\right\}}(\{u\}) Y_{k}^{i}(v) t_{i\left\{\beta^{\prime}\right\}}^{k\{\beta\}}(v,\{u\}) v^{\{\alpha\}} F_{\{\beta, \alpha\}}^{j}+\text { u.t. } \\
& =S^{\left\{\beta^{\prime}\right\}}(\{u\}) t_{i\left\{\beta^{\prime}\right\}}^{k\{\beta\}}(v,\{u\}) t_{k\left\{\alpha^{\prime}\right\}}^{* i\{\alpha\}}(v) v^{\left\{\alpha^{\prime}\right\}} F_{\{\beta, \alpha\}}^{j}+\text { u.t } \\
& =S^{\left\{\beta^{\prime}\right\}}(\{u\}) \bar{t}_{i\left\{\beta^{\prime}, \alpha^{\prime}\right\}}^{i\{\beta,}(v,\{u\}) v^{\left\{\alpha^{\prime}\right\}} F_{\{\beta, \alpha\}}^{j}+\text { u.t., }
\end{aligned}
$$

where

$$
\bar{t}_{i\left\{\beta^{\prime}, \alpha^{\prime}\right\}}^{i\{\beta, \alpha\}}(v,\{u\})=t_{i\left\{\beta^{\prime}\right\}}^{k\{\beta\}}(v,\{u\}) t_{k\left\{\alpha^{\prime}\right\}}^{* i\{\alpha\}}(v) .
$$


In the above,

$$
\begin{aligned}
t(v,\{u\}) & =r_{01}\left(v-u_{1}\right) r_{02}\left(v-u_{2}\right) \ldots r_{0 N}\left(v-u_{N}\right), \\
t^{*}(v) & =r_{01}^{*}(v) r_{02}^{*}(v) \ldots r_{0 l}^{*}(v) .
\end{aligned}
$$

and $r^{*}(u)$ is the $g l(1 \mid 1) R$-matrix defined from $R^{*}(u)$ by restricting the indices to $\alpha, \gamma=2,3$ and $\beta, \delta=a, b$ in (15) in analogy to $r(u)$. It is important to observe that the space $X$ is closed under the action of the elements $Y_{j}^{i}(u), i, j=2,3$ which generate a $g l(1 \mid 1)$-Yang-Baxter algebra. In fact

$$
Y_{j}^{i}(u) v^{\{\alpha\}}=t_{j\left\{\alpha^{\prime}\right\}}^{* i\{\alpha)}(u) v^{\left\{\alpha^{\prime}\right\}},
$$

which follows from the fact that the $Y_{j}^{i}(u), i, j=2,3$, act trivially on the vector $v^{1}$ in the sense

$$
\begin{aligned}
& Y_{2}^{2}(u) v^{1}=Y_{3}^{3}(u) v^{1}=v^{1} \\
& Y_{3}^{2}(u) v^{1}=Y_{2}^{3}(u) v^{1}=0 .
\end{aligned}
$$

An example of the action of $Y_{2}^{2}(u)$ and $Y_{3}^{3}(u)$ on the first pseudovacuum is illustrated below:

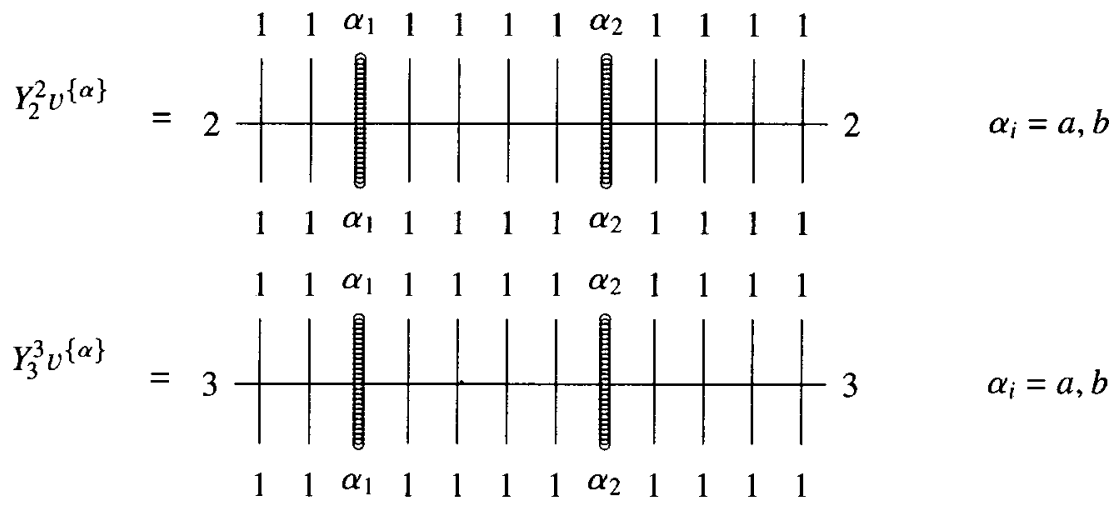

The contribution to the eigenvalues of the transfer matrix is

$$
\begin{aligned}
& Y_{2}^{2}(v) \Phi^{j}+(-1)^{1+[j]} Y_{3}^{3}(v) \Phi^{i} \\
& \quad=\sum_{i=2}^{3}(-1)^{[i]+[i][j]} \bar{t}_{i\left\{\beta^{\prime}, \alpha^{\prime}\right\}}^{i\{\beta\}}(v,\{u\}) S^{\left\{\beta^{\prime}\right\}}(\{u\}) v^{\left\{\alpha^{\prime}\right\}} F_{\{\beta, \alpha\}}^{j}+\text { u.t. }
\end{aligned}
$$

At this point we need to perform a second-level, or nested Bethe ansatz procedure to diagonalize the matrix

$$
\tau_{1}(v)_{\left\{\beta^{\prime}, \alpha^{\prime}\right\}}^{\{\beta, \alpha\}}=\sum_{i=2}^{3}(-1)^{[i]+\mid i][\{\beta, \alpha\}]} \bar{t}_{i\left\{\beta^{\prime}, \alpha^{\prime}\right\}}^{i\{\beta, \alpha\}}(v,\{u\}),
$$

where we have used the fact that $F_{\{\beta, \alpha\}}^{j}=0$ unless $[j]=[\{\beta, \alpha\}]$. The above matrix is simply the transfer matrix for a $g l(1 \mid 1)$ invariant system acting in the tensor product 
representation of $N$ copies of the vector representation with inhomogeneities $\{u\}$ and $l$ copies of the impurity sub-representation.

We diagonalize $\tau_{1}(v)$ in analogy to $\tau(v)(20)$ by constructing the nested monodromy matrix

$$
y(u)=\left(\begin{array}{ll}
y_{2}^{2}(u) & y_{2}^{3}(u) \\
y_{3}^{2}(u) & y_{3}^{3}(u)
\end{array}\right),
$$

satisfying

$$
r_{12}(u-v) y_{13}(u) y_{23}(v)=y_{23}(v) y_{13}(u) r_{12}(u-v) .
$$

From the above set of relations we will need the following:

$$
\begin{aligned}
& y_{2}^{2}(v) y_{2}^{3}(u)=a(u-v) y_{2}^{3}(u) y_{2}^{2}(v)-b(u-v) y_{2}^{3}(v) y_{2}^{2}(u), \\
& y_{3}^{3}(v) y_{2}^{3}(u)=-a(u-v) y_{2}^{3}(u) y_{3}^{3}(v)-b(v-u) y_{2}^{3}(v) y_{3}^{3}(u), \\
& y_{2}^{3}(v) y_{2}^{3}(u)=\frac{-a(u-v)}{a(v-u)} y_{2}^{3}(u) y_{2}^{3}(v) .
\end{aligned}
$$

Proceeding similarly as before, we look for eigenstates of the form

$$
\phi=y_{2}^{3}\left(\gamma_{1}\right) y_{2}^{3}\left(\gamma_{2}\right) \ldots y_{2}^{3}\left(\gamma_{M}\right) \theta
$$

with the second-level pseudovacuum $\theta$ given by

$$
\theta=S^{\{2\}}(\{u\}) v^{\{a\}} .
$$

It is an eigenstate of $y_{2}^{2}$ and $y_{3}^{3}$

$$
y_{2}^{2}(v) \theta=\prod_{i=1}^{N} a\left(v-u_{i}\right) \theta, \quad y_{3}^{3}(v) \theta=\left[w^{*}(v)\right]^{l} \theta .
$$

Using (30), (31) it follows that

$$
\tau_{1}(v) \phi=\Lambda_{1}(v) \phi+\text { u.t. }
$$

with

$$
\Lambda_{1}(v)=\prod_{i=1}^{N} a\left(v-u_{i}\right) \prod_{k=1}^{M} a\left(\gamma_{k}-v\right)-\left[w^{*}(v)\right]^{I} \prod_{k=1}^{M} a\left(\gamma_{k}-v\right) .
$$

The unwanted terms cancel provided the parameters $\gamma_{k}$ satisfy the Bethe ansatz equations (BAE)

$$
\prod_{i=1}^{N} a\left(\gamma_{k}-u_{i}\right)=\left[w^{*}\left(\gamma_{k}\right)\right]^{l}, \quad k=1,2, \ldots, M .
$$

Combining this result with Eq. (25) we obtain for the eigenvalues of the transfer matrix (11)

$$
\Lambda(v)=a(v)^{L} \prod_{i=1}^{N} a\left(u_{i}-v\right)+\Lambda_{1}(v) .
$$


Cancellation of the unwanted terms in (25), (27) leads to a second set of BAE:

$$
a\left(u_{h}\right)^{L} \prod_{i=1}^{N} \frac{a\left(u_{i}-u_{h}\right)}{a\left(u_{h}-u_{i}\right)}=-\prod_{k=1}^{M} a\left(\gamma_{k}-u_{h}\right), \quad h=1,2, \ldots, N
$$

Using (14), (16) and making the change of variables

$$
\begin{aligned}
& u_{h} \rightarrow i u_{h}+1 \\
& \gamma_{k} \rightarrow i \gamma_{k}+2
\end{aligned}
$$

we obtain

$$
\begin{aligned}
& \left(\frac{u_{h}+i}{u_{h}-i}\right)^{L}=\prod_{k \neq h}^{N} \frac{u_{h}-u_{k}+2 i}{u_{h}-u_{k}-2 i} \prod_{\delta=1}^{M} \frac{u_{h}-\gamma_{\delta}-i}{u_{h}-\gamma_{\delta}+i}, \quad h=1, \ldots, N=L+1-n_{\uparrow}, \\
& \prod_{h=1}^{N} \frac{\gamma_{\delta}-u_{h}+i}{\gamma_{\delta}-u_{h}-i}=\left(\frac{\gamma_{\delta}-i \alpha}{\gamma_{\delta}+i \alpha}\right)^{l}, \quad \delta=1, \ldots, M=L+2-n_{\uparrow}-n_{\downarrow} .
\end{aligned}
$$

In the absence of impurities (limit $l \rightarrow 0$ ) we recover the form of the BAE first derived by Sutherland [5] and later by Sarkar [7] for the usual $t-J$ model. The basic procedure to solve (37), (38) is to adopt the string conjecture, which means that the $u_{h}$ appear as strings and all roots $\gamma_{\delta}$ are real,

$$
\begin{aligned}
u_{\alpha j}^{n} & =u_{\alpha}^{n}+i(n+1-2 j), \quad j=1, \ldots, n, \quad \alpha=1, \ldots, N_{n}, \quad n=1,2,3, \ldots, \\
\gamma_{\delta} & =\text { real }, \quad \delta=1,2,3, \ldots, M,
\end{aligned}
$$

where $u_{\alpha}^{n}$ is the position of the centre of the string on the real $u$-axis. The number of $n$-strings $N_{n}$ satisfy the relation

$$
N=\sum_{n} n N_{n}
$$

Applying the string conjecture (39) in (37) and (38) and taking the product over $j$ in (37) and (38) we obtain

$$
\begin{aligned}
e^{L}\left(\frac{u_{\alpha}^{n}}{n}\right) & =\prod_{m} \prod_{\beta} E_{n m}\left(u_{\alpha}^{n}-u_{\beta}^{n l}\right) \prod_{\delta}^{M} e^{-1}\left(\frac{u_{\alpha}^{n}-\gamma_{\delta}}{n}\right), \\
\prod_{n} \prod_{\alpha} e\left(\frac{\gamma_{\delta}-u_{\alpha}^{n}}{n}\right) & =e^{-l}\left(\frac{\gamma_{\delta}}{\alpha}\right),
\end{aligned}
$$

where $e(x)=(x+i) /(x-i)$ and 


$$
E_{n m}(x)=\left\{\begin{array}{l}
e\left(\frac{x}{|n-m|}\right) e^{2}\left(\frac{x}{|n-m|+2}\right) \ldots \\
\quad \times e^{2}\left(\frac{x}{n+m-2}\right) e\left(\frac{x}{n+m}\right), \quad n \neq m \\
e^{2}\left(\frac{x}{2}\right) e^{2}\left(\frac{x}{4}\right) \ldots e^{2}\left(\frac{x}{2 n-2}\right) e^{2}\left(\frac{x}{2 n}\right), \quad n=m
\end{array}\right.
$$

Taking the logarithm of these equation and using that $\ln (e(x))=(2 / i) \arctan (x)-$ $\ln (-1)$, we get

$$
\begin{aligned}
L \theta\left(\frac{u_{\alpha}^{n}}{n}\right)-\sum_{m} \sum_{\beta=1}^{N_{m}} \Theta_{n m}\left(u_{\alpha}^{n}-u_{\beta}^{m}\right)+\sum_{\delta=1}^{M} \theta\left(\frac{u_{\alpha}^{n}-\gamma_{\delta}}{n}\right) & =2 \pi I_{\alpha}^{n}, \\
\sum_{n} \sum_{\alpha=1}^{N_{n}} \theta\left(\frac{\gamma_{\delta}-u_{\alpha}^{n}}{n}\right)+l \theta\left(\frac{\gamma_{\delta}}{\alpha}\right) & =2 \pi J_{\delta},
\end{aligned}
$$

where $\theta(x)=2 \arctan (x)$ and

$$
\Theta_{n m}(x)=\left\{\begin{array}{l}
\theta\left(\frac{x}{|n-m|}\right)+2 \theta\left(\frac{x}{|n-m|+2}\right)+\ldots \\
\quad+2 \theta\left(\frac{x}{n+m-2}\right)+\theta\left(\frac{x}{n+m}\right), \quad n \neq m \\
2 \theta\left(\frac{x}{2}\right)+2 \theta\left(\frac{x}{4}\right)+\ldots+2 \theta\left(\frac{x}{2 n-2}\right)+\theta\left(\frac{x}{2 n}\right), \quad n=m
\end{array}\right.
$$

Hence the solutions of (37) and (38) are parametrized in terms of the numbers $I_{\alpha}^{n}$ and $J_{\delta}$. The numbers $I_{\alpha}^{n}$ and $J_{\delta}$ are limited to the intervals

$$
\begin{aligned}
& \left|I_{\alpha}^{n}\right| \leqslant I_{\max }^{n}=\frac{1}{2}\left(L+M-\sum_{m} t_{m m} N_{m}-1\right), \\
& \left|J_{\delta}\right| \leqslant J_{\max }=\frac{1}{2}\left(\sum_{n} N_{n}+l-2\right),
\end{aligned}
$$

where $t_{m m}=2 \min (n, m)-\delta_{n, m}$.

As was shown in Refs. [8,9], two other forms of the Bethe ansatz exist which are obtained by choosing a different grading for the indices of the $g l(2 \mid 1)$ generators. Recall that the above calculations were performed with the choice

$$
[1]=[2]=0, \quad[3]=1 .
$$

Choosing

$$
[1]=1, \quad[2]=[3]=0
$$

yields the eigenvalue expression 


$$
\begin{aligned}
A(v)= & -[-w(v)]^{L}\left[g^{*}(v)\right]^{l} \prod_{i=1}^{N} a\left(v-u_{i}\right) \\
& +\left[b^{*}(v)\right]^{l}\left(\prod_{i=1}^{N} a\left(v-u_{i}\right) \prod_{k=1}^{M} a\left(\gamma_{k}-v\right)+\prod_{k=1}^{M} a\left(v-\gamma_{k}\right)\right)
\end{aligned}
$$

subject to the BAE

$$
\begin{aligned}
{\left[-w\left(u_{i}\right)\right]^{L}\left(\frac{g^{*}\left(u_{i}\right)}{b^{*}\left(u_{i}\right)}\right)^{l} } & =\prod_{k=1}^{M} a\left(\gamma_{k}-u_{i}\right), \quad i=1,2, \ldots, N, \\
\prod_{k=1}^{M} \frac{a\left(\gamma_{h}-\gamma_{k}\right)}{a\left(\gamma_{k}-\gamma_{h}\right)} & =-\prod_{i=1}^{N} a\left(\gamma_{h}-u_{i}\right), \quad h=1,2, \ldots, M .
\end{aligned}
$$

In the limit $l \rightarrow 0$ we recover Lai's form of the BAE [4] (see also Ref. [6]). Alternatively, choosing

$$
[1]=[3]=0, \quad[2]=1
$$

yields the eigenvalue expression

$$
\begin{aligned}
\Lambda(v)= & a(v)^{L} \prod_{i=1}^{N} a\left(u_{i}-v\right)+\left[b^{*}(v)\right]^{l} \prod_{k=1}^{M} a\left(v-\gamma_{k}\right) \\
& -\left[f^{*}(v)\right]^{l} \prod_{i=1}^{N} a\left(u_{i}-v\right) \prod_{k=1}^{M} a\left(v-\gamma_{k}\right)
\end{aligned}
$$

with the BAE

$$
\begin{aligned}
a\left(u_{i}\right)^{L} & =\left[f^{*}(v)\right]^{l} \prod_{k=1}^{M} a\left(u_{i}-\gamma_{k}\right), \quad i=1,2, \ldots, N, \\
1 & =\left(\frac{f^{*}\left(\gamma_{k}\right)}{b^{*}\left(\gamma_{k}\right)}\right)^{l} \prod_{i=1}^{N} a\left(u_{i}-\gamma_{k}\right), \quad k=1,2, \ldots, M .
\end{aligned}
$$

Finally, from the definition of the Hamiltonian (12) we see that the energies are given by

$$
E=-\left.2 \frac{d}{d v} \ln \left(v^{L} \Lambda(v)\right)\right|_{t^{\prime}=0}
$$

Using the eigenvalue expression (35) we obtain

$$
E=L-4 \sum_{i=1}^{N} \frac{1}{1+u_{i}^{2}}
$$

where the $u_{i}$ are solutions to Eqs. (37), (38). 


\section{Highest weight property}

Next we wish to show that the eigenstates constructed in the previous section are in fact highest weight states with respect to the underlying supersymmetry algebra $g l(2 \mid 1)$. The highest weight property of the Bethe states has been proved for many models, such as the Heisenberg chain [23] and its generalized version [28], the Kondo model [26], the usual $t-J$ model [9], the Hubbard chain [24] and its $g l(2 / 2)$ extension [25]. In all of these examples the Bethe ansatz was performed using a unique reference state. However, it was shown recently that the highest weight property can also hold for cases where the Bethe ansatz solution is obtained from a subspace of reference states [16].

Let us begin by considering

$$
E_{3}^{2} \Phi^{j}=\sum_{\{\beta, \alpha\}} E_{3}^{2} S^{\{\beta\}}(\{u\}) v^{\{\alpha\}} F_{\{\beta, \alpha\}}^{j} .
$$

By means of the nesting procedure we know that the coefficients $F_{\{\beta, \alpha\}}^{j}$ are such that we have the following identification of states:

$$
S^{\{\beta\}}(\{u\}) v^{\{\alpha\}} F_{\{\beta, \alpha\}}^{j}=y_{2}^{3}\left(\gamma_{1}\right) y_{2}^{3}\left(\gamma_{2}\right) \ldots y_{2}^{3}\left(\gamma_{M}\right) w
$$

for a suitable solution of the BAE. By comparing Eqs. (6), (18), (29) it is possible to determine algebraic relations between the elements of the Yangian algebra and the supersymmetry algebra. For our purposes we need the following:

$$
\left[E_{3}^{2}, y_{2}^{3}(u)\right]_{\beta}^{\alpha}=-y_{2}^{2}(u)_{\beta}^{\alpha}+y_{3}^{3}(u)_{\beta}^{\alpha}(-1)^{\lfloor\alpha]} .
$$

Noting that $E_{3}^{2} w=0$ it is evident that we may write

$$
E_{3}^{2} y_{2}^{3}\left(\gamma_{1}\right) \ldots y_{2}^{3}\left(\gamma_{M}\right) w=\sum_{h=1}^{M} x_{h} X_{h}
$$

with

$$
X_{h}=y_{2}^{3}\left(\gamma_{1}\right) \ldots y_{2}^{3}\left(\gamma_{h-1}\right) y_{2}^{3}\left(\gamma_{h+1}\right) \ldots y_{2}^{3}\left(\gamma_{M}\right) w
$$

and the $x_{h}$ some yet to be determined coefficients. To find $x_{h}$ we write

$$
y_{2}^{3}\left(\gamma_{1}\right) \ldots y_{2}^{3}\left(\gamma_{M}\right) w=\prod_{j=1}^{h-1} \frac{-a\left(\gamma_{h}-\gamma_{j}\right)}{a\left(\gamma_{j}-\gamma_{h}\right)} y_{2}^{3}\left(\gamma_{h}\right) X_{h},
$$

where we have used Eq. (32). Now by using the relations (30), (31), (50) and looking only for those terms which give a vector proportional to $X_{h}$, we find that

$$
\begin{aligned}
x_{h}= & \prod_{j=1}^{h-1} \frac{-a\left(\gamma_{h}-\gamma_{j}\right)}{a\left(\gamma_{j}-\gamma_{h}\right)} \\
& \times\left(\left[w^{*}\left(\gamma_{h}\right)\right]^{l} \prod_{k \neq h}^{M} a\left(\gamma_{k}-\gamma_{h}\right)-\prod_{i=1}^{N} a\left(\gamma_{h}-u_{i}\right) \prod_{k \neq h}^{M} a\left(\gamma_{k}-\gamma_{h}\right)\right),
\end{aligned}
$$


which vanishes because of Eq. (34). Thus we see that

$$
E_{3}^{2} \Phi^{j}=0 \text {. }
$$

Next we consider the action of $E_{2}^{1}$ on $\Phi^{j}$. Using Eqs. (6), (19), (18) we find the commutation relation

$$
\left[E_{2}^{1}, Y_{1}^{\alpha}(u)\right]=\delta_{2}^{\alpha} Y_{1}^{1}(u)-Y_{2}^{\alpha}(u) .
$$

As before, since $E_{2}^{1} v^{\{\alpha\}}=0$ we can write the general expression

$$
E_{2}^{1} \Phi^{j}=\sum_{h, \beta} z_{h, \beta} Z_{h, \beta}
$$

where

$$
\left.\left.Z_{h, \beta}=S^{\left\{\beta_{h}^{-}\right\}}\left(\left\{u_{h}^{-}\right)\right\}\right) S^{\left\{\beta_{h}^{+}\right\}}\left(\left\{u_{h}^{+}\right)\right\}\right) v^{\{\alpha\}} F_{\{\beta, \alpha\}}^{j}
$$

and for any vector $\{w\}$ we have

$$
\left\{w_{h}^{-}\right\}=\left(w_{1}, w_{2}, \ldots, w_{h-1}\right), \quad\left\{w_{h}^{+}\right\}=\left(w_{h+1}, \ldots, w_{N}\right) .
$$

To calculate $z_{h, \beta}$ we begin by writing

$$
\begin{aligned}
\Phi^{j}= & S^{\left\{\beta_{h}^{-}\right\}}\left(\left\{u_{h}^{-}\right\}\right) Y_{1}^{\beta_{h}}\left(u_{h}\right) S^{\left\{\beta_{h}^{+}\right\}}\left(\left\{u_{h}^{+}\right\}\right) v^{\{\alpha\}} F_{\{\beta, \alpha\}}^{j} \\
= & \prod_{i=1}^{h-1} a\left(u_{i}-u_{h}\right)^{-1} t_{\gamma\left\{\gamma_{h}^{-}\right\}}^{\beta_{h}\left\{\beta_{h}^{-}\right\}}\left(-u_{h},\left\{-u_{h}^{-}\right\}\right) \\
& \times Y_{1}^{\gamma}\left(u_{h}\right) S^{\left\{\gamma_{h}^{-}\right\}}\left(\left\{u_{h}^{-}\right\}\right) S^{\left\{\beta_{h}^{+}\right\}}\left(\left\{u_{h}^{+}\right\}\right) v^{\{\alpha\}} F_{\{\beta, \alpha\}}^{j},
\end{aligned}
$$

where we have used the relation (24). Now applying Eq. (51) and using the relations (22), (23) to determine the terms which give a vector proportional to $Z_{h, \beta}$ we find that

$$
z_{h, \beta}=\delta_{2}^{\beta_{h}}\left(a\left(u_{h}\right)^{L} \prod_{i \neq h}^{N} a\left(u_{i}-u_{h}\right)-\prod_{i \neq h}^{N} a\left(u_{h}-u_{i}\right) \prod_{k=1}^{M} a\left(\gamma_{k}-u_{h}\right)\right),
$$

which vanishes as a result of Eq. (36). We then conclude that

$$
E_{2}^{\mathrm{l}} \Phi^{j}=0
$$

Finally, using the fact that

$$
E_{3}^{1}=\left[E_{2}^{1}, E_{3}^{2}\right]
$$

it is deduced that

$$
E_{3}^{\mathrm{l}} \Phi^{j}=0,
$$

which completes the proof that the Bethe states are $g l(2 \mid 1)$ highest weight states. We observe that this property can also be proved for the other two choices of gradings in a similar way. 
By acting with the $g l(2 \mid 1)$ lowering operators on the Bethe states additional states are obtained which are necessarily eigenstates of the Hamiltonian as a consequence of the $g l(2 \mid 1)$ invariance. Before proceeding to counting the number of eigenstates, it is important to assert that each of the Bethe states belongs to an irreducible submodule of the full tensor product space. Recall that unlike the case of Lie algebras, there exist representations of Lie superalgebras which can be indecomposable but not irreducible [29]. However, this situation does not occur in the decomposition of the tensor representation here, which follows from the fact that both the fundamental representation and the family of four-dimensional representations with $\alpha>0$ belong to the class of type I unitary (or star) representations defined in [29] and classified in [30]. A general theorem states [29] that tensor products of type I unitary representations are always completely reducible into representations which are also of type I. Moreover, since the module which acts for the impurity spaces is typical it follows from Ref. [31] (see Proposition 2) that all the modules occurring in the decomposition of the tensor product space are in fact typical.

\section{Completeness of the Bethe states}

In this section we show how to construct a complete set of eigenvectors for the $t-J$ Hamiltonian for an arbitrary chain of length $L$ with $l$-impurities. This is obtained by combining the Bethe ansatz equations with the supersymmetry of the model.

From the previous section we know that all collections $\left\{I_{\alpha}^{n}, J_{\delta}\right\}$ where the $I$ 's and $J$ 's are pairwise different specify all the Bethe vectors. The number of admissible values

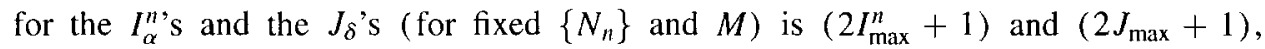
respectively. Taking into account that many different string configurations $N_{n}$ give the same number of roots $N$, the number of possible Bethe vectors for fixed $N, M$ is given by

$$
Z(N, M)=\sum_{\left\{N_{n}\right\}}\left(\begin{array}{c}
2 J_{\max }+1 \\
M
\end{array}\right) \prod_{n}\left(\begin{array}{c}
2 I_{\max }^{n}+1 \\
N_{n}
\end{array}\right)
$$

where the sum over $\left\{N_{n}\right\}$ is constrained to $\sum_{n} n N_{n}=N$. It is convenient to introduce the quantity $q=\sum_{n} N_{n}$. Then we can write this sum as follows:

$$
Z(N, M)=\sum_{q=0}^{N}\left(\begin{array}{c}
q+l-1 \\
M
\end{array}\right) \sum_{\left\{N_{n}\right\}} \prod_{n}\left(\begin{array}{c}
L-\sum_{m} t_{n m} N_{m}+M \\
N_{n}
\end{array}\right),
$$

where the inner sum is constrained to fixed values of $N$ and $q$. This expression resembles the one calculated by Bethe in the isotropic Heisenberg model [22,23] and can be simplified to

$$
Z(N, M)=\sum_{q=0}^{N} \frac{L+M-2 N+1}{L+M-N+1}\left(\begin{array}{c}
q+l-1 \\
M
\end{array}\right)\left(\begin{array}{c}
L+M-N+1 \\
q
\end{array}\right)\left(\begin{array}{c}
N-1 \\
q-1
\end{array}\right) .
$$


The total number of Bethe vectors is obtained by summing $Z(N, M)$ over all $N, M$. From the fact that the Bethe vectors are highest weight vectors, by acting with the $g l(2 \mid 1)$ lowering operators $E_{j}^{i}(i \geqslant j)$ on the Bethe states we obtain additional states. Each Bethe state (with fixed $N, M$ ) is the highest weight vector in an irreducible multiplet of dimension [29]

$$
d(N, M)=8\left(S_{z}+1 / 2\right)=4(L-2 N+M+1) .
$$

Notice that here, in contrast to the usual $t-J$ model [9] only typical $g l(2 \mid 1)$ multiplets occur as discussed in the previous section. With these considerations, the total number of eigenvectors is

$$
Z=\sum_{M=0}^{L+2 l} \sum_{N=M-l}^{\frac{L+M}{2}} d(N, M) Z(N, M) .
$$

Above the ranges of $M$ and $N$ are obtained by demanding that the magnetization $S_{z}=$ $\frac{1}{2}\left(n_{\uparrow}-n_{\downarrow}\right)=\frac{1}{2}(L-2 N+M)$ and the number of electron $Q=n_{\uparrow}+n_{\downarrow}=L+2 l-M$ are restricted to intervals $0 \leqslant S_{z} \leqslant \frac{L+l}{2}$ and $0 \leqslant Q \leqslant L+2 l$,

$$
\begin{aligned}
Z= & \sum_{M=0}^{L+2 l} \sum_{N=M-l}^{\frac{L 1 M}{2}} 4(L-2 N+M+1) \frac{L+M-2 N+1}{L+M-N+1} \\
& \times \sum_{q=0}^{N}\left(\begin{array}{c}
q+l-1 \\
M
\end{array}\right)\left(\begin{array}{c}
L+M-N+1 \\
q
\end{array}\right)\left(\begin{array}{c}
N-1 \\
q-1
\end{array}\right) .
\end{aligned}
$$

Using the identity

$$
\left(\begin{array}{c}
q+l-1 \\
M
\end{array}\right)=\sum_{k=0}^{l-1}\left(\begin{array}{c}
l-1 \\
k
\end{array}\right)\left(\begin{array}{c}
q \\
M-k
\end{array}\right), \quad l \geqslant 1
$$

Eq. (55) can be written as

$$
\begin{aligned}
Z= & \sum_{k=0}^{l-1}\left(\begin{array}{c}
l-1 \\
k
\end{array}\right) \sum_{M=0}^{L+2 l} \sum_{N=M-l}^{\frac{L+M}{2}} 4(L-2 N+M+1) \frac{L+M-2 N+1}{L+M-N+1} \\
& \times \sum_{q=0}^{N}\left(\begin{array}{c}
q \\
M-k
\end{array}\right)\left(\begin{array}{c}
L+M-N+1 \\
q
\end{array}\right)\left(\begin{array}{c}
N-1 \\
q-1
\end{array}\right) .
\end{aligned}
$$

Employing some combinatorics, we find

$$
\begin{aligned}
Z= & \sum_{k=0}^{l-1}\left(\begin{array}{c}
l-1 \\
k
\end{array}\right) \sum_{M=0}^{L+2 l} \sum_{N=M-l}^{\frac{L+M}{2}} 4(L-2 N+M+1) \\
& \times \frac{L+M-2 N+1}{L+M-N+1}\left(\begin{array}{c}
L+M-N+1 \\
M-k
\end{array}\right)\left(\begin{array}{c}
L+k \\
N-M+k
\end{array}\right) .
\end{aligned}
$$

After rearrangement this expression can be rewritten as 


$$
\begin{aligned}
Z= & \sum_{k=0}^{l-1}\left(\begin{array}{c}
l-1 \\
k
\end{array}\right) \sum_{M=0}^{L+2 l} \sum_{N=M-l}^{\frac{L+M}{2}} 4(L-2 N+M+1) \\
& \times\left\{\left(\begin{array}{c}
L+M-N+1 \\
M-k
\end{array}\right)\left(\begin{array}{c}
L+k \\
N-M+k
\end{array}\right)-\left(\begin{array}{c}
N \\
M-k
\end{array}\right)\left(\begin{array}{c}
L+k \\
N-1
\end{array}\right)\right\} .
\end{aligned}
$$

Making the substitution $N \rightarrow x+M-l$ we obtain

$$
\begin{aligned}
Z= & \sum_{k=0}^{l-1}\left(\begin{array}{c}
l-1 \\
k
\end{array}\right) \sum_{M=0}^{L+2 l} \sum_{x=0}^{L-M}+1 \\
& \times\left\{\left(\begin{array}{c}
L+l-x+1 \\
M-k
\end{array}\right)\left(\begin{array}{c}
L+k \\
x-l+k
\end{array}\right)-\left(\begin{array}{c}
x+M-l \\
M-k
\end{array}\right)\left(\begin{array}{c}
L+k \\
x+M-l-1
\end{array}\right)\right\},
\end{aligned}
$$

which, after some manipulation, turns out to be

$$
\begin{aligned}
& Z=4 \sum_{k=0}^{l-1}\left(\begin{array}{c}
l-1 \\
k
\end{array}\right) \sum_{M=0}^{L+2 l}(M-k+1)\left\{\sum_{x=0}^{\frac{L-M}{2}+l}\left(\begin{array}{c}
L+l-x+1 \\
M-k+1
\end{array}\right)\left(\begin{array}{c}
L+k \\
x-l+k
\end{array}\right)\right. \\
& \left.+\sum_{x=0}^{\frac{L-M}{2}+l}\left(\begin{array}{c}
x+M-l \\
M-k+1
\end{array}\right)\left(\begin{array}{c}
L+k \\
x+M-l-1
\end{array}\right)\right\} \\
& -4 \sum_{k=0}^{l-1}\left(\begin{array}{c}
l-1 \\
k
\end{array}\right) \sum_{M=0}^{L+2 l}(L+k)\left\{\sum_{x=0}^{\frac{L-M}{2}+l}\left(\begin{array}{c}
L+l-x+1 \\
M-k
\end{array}\right)\left(\begin{array}{c}
L+k-1 \\
x-l+k-1
\end{array}\right)\right. \\
& \left.+\sum_{x=0}^{\frac{L-M}{2}+l}\left(\begin{array}{c}
L+k-1 \\
x+M-l-1
\end{array}\right)\left(\begin{array}{c}
x+M-l \\
M-k
\end{array}\right)\right\}
\end{aligned}
$$

Substituting $x \rightarrow L-x-M+2 l+1$ in the second and fourth terms of the equation above we get

$$
\begin{aligned}
Z= & 4 \sum_{k=0}^{l-1}\left(\begin{array}{c}
l-1 \\
k
\end{array}\right) \sum_{M=0}^{L+2 l}(M-k+1) \sum_{x=0}^{L-M+2 l+1}\left(\begin{array}{c}
L-x+l+1 \\
M-k+1
\end{array}\right)\left(\begin{array}{c}
L+k \\
x-l+k
\end{array}\right) \\
& -4 \sum_{k=0}^{l-1}\left(\begin{array}{c}
l-1 \\
k
\end{array}\right) \sum_{M=0}^{L+2 l}(L+k) \sum_{x=0}^{L-M+2 l+1}\left(\begin{array}{c}
L-x+l+1 \\
M-k
\end{array}\right)\left(\begin{array}{c}
L+k-1 \\
x-l+k-1
\end{array}\right) .
\end{aligned}
$$

We now find

$$
Z=-4 \sum_{k=0}^{l-1}\left(\begin{array}{c}
l-1 \\
k
\end{array}\right) \sum_{M=0}^{L+2 l}\left(\begin{array}{c}
L+k \\
M-k
\end{array}\right) \frac{d}{d P}\left[\sum_{x=0}^{L-M+2 k}\left(\begin{array}{c}
L-M+2 k \\
x-l+k
\end{array}\right) P^{x-l-L-1}\right]_{P=1}
$$




$$
+4 \sum_{k=0}^{l-1}\left(\begin{array}{c}
l-1 \\
k
\end{array}\right) \sum_{M=0}^{L+2 l}\left(\begin{array}{c}
L+k \\
M-k
\end{array}\right) \frac{d}{d P}\left[\sum_{x=0}^{L-M+2 k}\left(\begin{array}{c}
L-M+2 k \\
x-l+k-1
\end{array}\right) P^{x-L-l-1}\right]_{P=1}
$$

and using the binomial formula we obtain

$$
Z=4 \frac{d}{d P}\left[\sum_{k=0}^{l-1}\left(\begin{array}{c}
l-1 \\
k
\end{array}\right)\left(1-P^{-1}\right) P^{-k-L} \sum_{M=0}^{L+2 l}\left(\begin{array}{c}
L+k \\
M-k
\end{array}\right)(1+P)^{L-M+2 k}\right]_{P=1} .
$$

Above the sum over $M$ can be performed giving

$$
Z=4 \frac{d}{d P}\left[\left(1-P^{-1}\right) \sum_{k=0}^{l-1}\left(\begin{array}{c}
l-1 \\
k
\end{array}\right) P^{-k-L}(2+P)^{L+k}\right]_{P=1} .
$$

Now the sum over $k$ can be evaluated yielding

$$
4 \frac{d}{d P}\left[\left(1-P^{-1}\right) P^{-L-l+1}(2+P)^{L}(2+2 P)^{l-1}\right]_{P=1},
$$

which finally leads to

$$
Z=3^{L} 4^{l}
$$

Thus we have shown that the number of eigenvectors of the $t-J$ Hamiltonian with $l$ impurities is $3^{L} 4^{\prime}$, which is precisely the number of states in the Hilbert space of a chain of length $L$ with $l$ impurities. We point out that this is the first time that completeness of the Bethe vectors has been demonstrated for an impurity electronic model.

\section{Acknowledgements}

J.L. thanks the Fundação de Amparo a Pesquisa do Estado do Rio Grande do Sul and Australian Research Council for financial support. He also thanks the Instituto de Física da UFRGS for their kind hospitality. A.F. and A.P.T. thank CNPq-Conselho Nacional de Desenvolvimento Científico e Tecnológico for financial support.

\section{References}

[1] N. Andrei and H. Johannesson, Phys. Lett. A 100 (1984) 108.

[2] P. Schmitteckert, P. Schwab and U. Eckern, Europhys. Lett. 30 (1995) 543.

[3] H-P. Eckle, A. Punnoose and R. Römer, Europhys. Lett. 39 (1997) 293.

[4] C.K.Lai, J. Math. Phys 15 (1974) 1675.

[5] B. Sutherland, Phys. Rev. B 12 (1975) 3795.

16] P. Schlottmann, Phys. Rev. B 36 (1987) 5177 
[7] S.Sarkar, J. Phys. A 24 (1991) 1137.

$18 \mid$ F.H.L. Essler and V.E. Korepin, Phys. Rev. B 46 (1992) 9147.

[9] A. Foerster and M. Karowski, Phys. Rev. B 46 (1992) 9234; Nucl. Phys. B 396 ( 1993 ) 611.

[10] G. Bedürftig, F.H.L. Essler and H. Frahm, Phys. Rev. Lett. 77 (1996) 5098; Nucl. Phys. B 498 (1997) 697.

[11] A.J. Bracken, M.D. Gould, J. Links and Y.-Z. Zhang, Phys. Rev. Lett. 74 (1995) 2768.

[12] J. Abad and M. Ríos, Phys. Rev. B 53 (1996) 14000.

[13] J. Abad and M. Ríos, J. Phys. A 30 (1997) 5887.

[14] M.P. Pfannmuller and H. Frahm, J. Phys. A 30 (1997) L543.

[15] J. Abad and M. Ríos, Exact solution of a electron system combining two different $t-J$ models, condmat/9806106.

[16] J. Links and A. Foerster, J. Phys. A 32 (1999) 147.

| $17 \mid$ M. J. Martins, Integrable mixed vertex models from braid-monoid algebra, preprint UFSCAR-TH-98-12.

|18| P.-A. Bares, Exact results for a one dimensional $t-J$ model with impurities, cond-mat/9412011.

[19] P. Schlottmann and A. A. Zvyagin, Phys. Rev. B 55 (1997) 5027; B 56 (1997) 13989.

|20| Y. Wang, J.-H. Dai, Z.-N. Hu and F.-C. Pu, Phys. Rev. Lett. 79 (1997) 1901; Z.-N. Hu, F.-C. Pu and Y. Wang, J. Phys. A 31 (1998) 5241.

[21] H.-Q. Zhou and M.D. Gould, Algebraic Bethe ansatz for integrable Kondo impurities in the onedimensional supersymmetric $t-J$ model, cond-mat/9809055;

H.-Q. Zhou, X.-Yu Ge, J. Links and M. D. Gould, Graded reflection equations algebras and integrable Kondo impurities in the one-dimensional $t-J$ model, cond-mat/9809056.

[22] H. Bethe, Z. Phys. 71 (1931) 205.

[23] L.D. Faddeev and L. Takhtajan, Zap. Nauch. Semin. LOMI 109 (1981) 134.

[24] F. Essler, V. Korepin and K. Schoutens, Phys. Rev. Lett. 67 (1991) 3848; Nucl. Phys. B 372 (1992) 559; B 384 (1992) 431.

[25] K. Schoutens, Nucl. Phys. B 413 (1994) 675.

[26] A.N. Kirillov, J. Sov. Math. 34 (1985) 2298.

127| G.W. Delius, M.D. Gould, J.R. Links and Y.-Z. Zhang, Int. J. Mod. Phys. A 10 (1995) 3259.

|28| A.N. Kirillov, J. Sov, Math. 36 (1987) 115.

[29] M. Scheunert, W. Nahm and V. Rittenberg, J. Math. Phys. 18 (1977) 146.

[30] M.D. Gould and R.B. Zhang, J. Math. Phys. 31 (1990) 1524.

[3I] M.D. Gould and J. Links, J. Phys. A 30 (1997) 1613. 